\title{
Análise da complexidade do saldo de radiação em uma floresta sazonalmente inundada no norte do pantanal
}

\author{
Analysis of the complexity of net radiation in a seasonal flooded forest in the northern Pantanal
}

Geison Jader Mello', Sérgio Wagner Gripp da Silveira², Raphael de Souza Rosa Gomes³, Carlo Ralph de Musis ${ }^{4}$, Sergio Roberto de Paulo ${ }^{5}$, Nadja Gomes Machado ${ }^{6}$ e Marcelo Sacardi Biudes ${ }^{7}$

\author{
1, 2, 3, 4, 5,6, 7. Programa de Pós-Graduação em Física Ambiental - Instituto de Física - Universidade Federal de Mato Grosso \\ ${ }^{6}$ Laboratório de Biologia da Conservação - Instituto Federal de Mato Grosso. Professora do Programa de Pós-Graduação em Física Ambiental - \\ Instituto de Física - Universidade Federal de Mato Grosso
}

\begin{abstract}
Resumo
O saldo de radiação em ecossistemas possui comportamento complexo e representa a energia disponível para os processos físicos e biológicos que ocorrem entre a superfície vegetada e a atmosfera. Sendo assim, o objetivo deste trabalho foi analisar a complexidade do saldo de radiação medido acima do dossel de uma floresta sazonalmente inundada no Norte do Pantanal, sob a ótica da teoria da complexidade. Para tanto, foram reconstruídos os atratores sazonalmente, estimando os expoentes dimensão de correlação $\left(\mathrm{D}_{2 \text { (chuvoso) }}=1,73 \pm 0,05\right.$ e $\left.\mathrm{D}_{2 \text { (seco) }}=1,36 \pm 0,18\right)$, dimensão embutida $(\mathrm{m}=7)$, taxa de recorrência $\left(\mathrm{RR}_{\text {(chuvoso) }}=0,144 \pm 0,004\right.$ e $\left.\mathrm{RR} \mathrm{(seco)}=0,169 \pm 0,002\right)$ e o determinismo $\left(\mathrm{DET}_{\text {(chuvoso) }}=0,896 \pm 0,013 \mathrm{e} \mathrm{DET}_{\text {(seco) }}=0,973 \pm 0,015\right)$ das séries de dados analisados. Os resultados evidenciam a existência de atratores estranhos de baixa dimensão fractal e sensíveis à sazonalidade do saldo de radiação.
\end{abstract}

Palavras-chave: atratores, gráficos de recorrência, dinâmica não linear.

\begin{abstract}
The ecosystem net radiation has a complex variation, and represents the energy available for physical and biological processes that occur between the vegetated surface and atmosphere. Therefore, the objective of this study was to analyze the complexity of net radiation measured above the canopy of a seasonal flooded forest in the northern Pantanal from the perspective of complexity theory. The attractors were seasonal reconstructed by the estimation of the exponents correlation dimension $\left(\mathrm{D}_{2 \text { (wet) }}=1,73 \pm 0,05\right.$ and $\left.\mathrm{D}_{2 \text { (dry) }}=1,36 \pm 0,18\right)$, embedded dimension $(\mathrm{m}=7)$, rate of recurrence $\left(\mathrm{RR}_{\text {(wet) }}=0,144 \pm 0,004\right.$ and $\left.\mathrm{RR}_{\text {(dry) }}=0,169 \pm 0,002\right)$ and determinism $\left(\mathrm{DET}_{\text {(wet) }}=0,896 \pm 0,013\right.$ and $\left.\mathrm{DET}_{(\mathrm{dry})}=0,973 \pm 0,015\right)$ data sets analyzed. The results showed the existence of strange attractors of low fractal dimension, and sensitive to the seasonality of radiation balance.
\end{abstract}

Keywords: attractors; recurrence plot; nonlinear dynamics. 


\section{INTRODUÇÃO}

A complexidade dos ecossistemas florestais (tropicais, inundados e/ou cerrado), dado o seu número e a variedade de seus subsistemas em interação, assim como os efeitos antropogênicos, torna a sua descrição um problema brasileiro extremamente significativo. (Gomes \& Varriale, 2004).

O Pantanal é a maior planície tropical de inundação sazonal do planeta, com aproximadamente 138 mil km², e abriga biodiversidade desproporcionalmente alta e influencia substancialmente o ciclo de carbono e outros gases do efeito estufa. O Pantanal distingue-se de outros biomas por apresentar fauna e flora peculiar que desempenha papel muito importante no saldo de radiação, na manutenção dos fluxos de energia e vapor de água, regulação da temperatura e estabilidade dinâmica do clima regional e global (Silva \& Abdon, 1998; Millenium Ecosystem Assesssment, 2005; Junk \& Nunes da Cunha, 2005).

Pesquisadores afirmam que alterações no ciclo hidrológico, observado pelo aumento dos picos de precipitação máxima, sugerem que a região está sendo afetada pelas mudanças climáticas (Diniz Fonseca, M.; Campelo Jr et al., 2008). Com baixa drenagem, o solo pantaneiro frequentemente prolonga o período de alagamento, limitando a estação seca de abril até setembro. A seca causa estresse hídrico nas plantas locais, regulando sua fenologia e produção de fitomassa (Junk, 2002). Há ainda rápida mudança na vegetação em campos sazonalmente inundados que também tem sido considerado um efeito de mudanças climáticas ocorridas em ciclos plurianuais (Arieira \& Nunes da Cunha, 2006). O Pantanal é suscetível a uma série de consequências ecológicas causadas pelo desmatamento, como o aumento da temperatura do ar, redução da precipitação e o prolongamento da seca pela ampliação das áreas destinadas à criação de gado e agricultura na região (Nobre et al., 1996; McClain, 2002; Costa \& Pires, 2010; Lewis et al., 2011, Lapola et al, 2011).

Com a troca da cobertura vegetal original por áreas de pastagens e cultivos, o fluxo de vapor de água para a atmosfera é alterado, bem como a refletividade do solo, afetando diretamente o saldo de radiação entre outros efeitos. Biomas tropicais são sistemas abertos, dinâmicos e complexos, o que os torna importantes para a avaliação de seus balanço de energia, no que diz respeito às estimativas da evapotranspiração (ET), aquecimento do ar e do solo, e ainda prever as suas contribuições com as mudanças climáticas locais e globais a médio e longo prazo (Pereira et al., 2002; IPCC,
2007, Motta et al., 2011).

Ainda não se compreende como essas alterações regionais no uso do solo podem afetar o ciclo hidrológico e o regime de chuvosos da região. Um dos motivos é a falta de séries históricas de dados micrometeorológicos medido in situ para detectar e monitorar estas possíveis mudanças no clima regional.

Embora haja estudos das relações entre radiação líquida e suas partições no Pantanal, ecossistemas são sistemas não determinísticos, não lineares, em que a influência de um estado anterior da dinâmica sobre o estado posterior não é diretamente proporcional, mas envolve laços de retroalimentação que podem influenciar o sistema globalmente (Souza \& Buckeridge, 2004). Além disso, a recorrência de estados, no sentido de que os estados arbitrários do sistema poderão estar próximos novamente com o avanço do tempo, é uma propriedade fundamental de sistemas dinâmicos determinísticos e é típico para sistemas não lineares (Marwan, 2007).

Sistemas abertos fora do equilíbrio são caracterizados por atratores, que são regiões restritas do espaço de estados para onde as trajetórias do sistema convergem. No caso de um sistema com dinâmica puramente aleatória, o atrator do sistema preenche o espaço de estados inteiramente, isto é, não há nenhum tipo de organização temporal, a dinâmica do sistema é totalmente imprevisível a curto ou longo prazo. Enquanto que em um sistema aberto e fora do equilíbrio sua dinâmica fica restrita a uma região do espaço de estados do atrator (Souza \& Buckeridge, 2004).

A importância da aplicação de ferramentas adequadas para se compreender os processos de troca de energia, matéria e momentum em floretas tropicais é destacada em pesquisas que abordam a natureza não linear dos dados ambientais, contribuindo para o entendimento e modelagem da interação solo-planta-atmosfera.

Embasado na teoria dos sistemas dinâmicos, várias pesquisas envolvendo séries temporais de diferentes variáveis ambientais mencionam a existência de atratores climáticos de baixa dimensão fractal e indicam o número mínimo de variáveis presentes na evolução da dinâmica do sistema (Ruelle \& Takens,1971; Nicolis \& Nicolis, 1984; Fraedrich, 1986; Lorenz, 1991; Waldrop, 1992; Povedo-Jaramillo \& Puente, 1993; Nicolis \& Prigogine, 1998; Xin et al., 2001; Gallego et al., 2001; Peitgen et al., 2004; Campanharo et al., 2008).

Por definição, um Gráfico de Recorrência (GR) é uma técnica avançada de análise de dados de sistemas não lineares por meio da visualização 
de gráficos formados por uma matriz quadrada, em que os elementos da matriz correspondem aos momentos em que um estado do sistema dinâmico se repete (colunas e linhas correspondem então a um determinado par de vezes). Tecnicamente, o GR revela todos os momentos em que a trajetória do espaço de fase do sistema dinâmico visita mais ou menos a mesma região no espaço de fase.

O estudo dos GRs dos sistemas dá uma boa introdução sobre a tipologia característica e a textura. No entanto, sua quantificação oferece uma maneira mais objetiva para a investigação do sistema considerado. As técnicas de quantificação para análise de dados, ou seja, a quantificação das medidas de complexidade dos GRs, têm se tornado muito popular num grupo crescente de cientistas de diversas áreas. Sendo assim, o objetivo deste trabalho foi analisar a complexidade do saldo de radiação medido acima do dossel de uma floresta sazonalmente inundada no Norte do Pantanal, sob a ótica da teoria da complexidade. Para tanto, foram utilizados GR através da representação bidimensional de trajetórias no espaço de fase (o que é vantajoso por se tratar de um sistema de alta dimensão), sendo possível a verificação dos padrões típicos que estão ligados ao comportamento específico do sistema; e as Medidas de Quantificação de Recorrência (MQR) para contabilização dos pontos e das diagonais, quantificando a complexidade pela taxa de recorrência (RR) e determinismo (DET) do sistema.

\section{Gráficos de Recorrência}

O gráfico de recorrência (GR) representa o número de vezes que o sistema recorre a um estado anterior, ou seja, em que uma trajetória visita a mesma região no espaço de fase. Essa trajetória no espaço de fase pode ser reconstruída a partir de uma única série temporal pela incorporação de tempos de atrasos $(\tau)$, segundo o teorema de Takens (1981). O GR é obtido após algumas etapas. Primeiramente, obtém-se (ou reconstrói-se) outras variáveis $\left\{\mathrm{X}_{\mathrm{t}}(\mathrm{t})\right\}$ (sendo $\mathrm{k}=1, \ldots, \mathrm{n}-1$ ) conhecendo somente $\mathrm{X}_{0}(\mathrm{t})$. Depois, desmembra-se a série temporal original $\mathrm{X}_{0}(\mathrm{t})$ em deslocamentos temporais sucessivos de defasagem fixa $\tau(\tau=\mathrm{m} \Delta \mathrm{t}$, onde $\mathrm{m}$ é um número inteiro e $\Delta$ t é um intervalo entre sucessivas amostras) para "n" pontos equidistantes do conjunto de dados. Isto é:

$$
\begin{aligned}
& \mathrm{X}_{0}: \quad \mathrm{X}_{0}\left(\mathrm{t}_{1}\right), \ldots, \mathrm{X}_{0}\left(\mathrm{t}_{\mathrm{N}}\right) \\
& \mathrm{X}_{1}: \quad \mathrm{X}_{0}\left(\mathrm{t}_{1}+\tau\right), \ldots, \mathrm{X}_{0}\left(\mathrm{t}_{\mathrm{N}}+\tau\right) \\
& : \\
& \mathrm{X}_{\mathrm{n}-1}: \mathrm{X}_{0}\left[\mathrm{t}_{1}+(\mathrm{n}-1) \tau\right], \ldots, \mathrm{X}_{0}\left[\mathrm{t}_{\mathrm{N}}+(\mathrm{n}-1) \tau\right]
\end{aligned}
$$

em que a melhor escolha de $\tau$ deve apresentar uma independência linear entre a série original e as séries defasadas, ou seja, a menor correlação, no intuito de obter a maior informação possível do sistema estudado.

Eckmann et al. (1987) introduziram uma ferramenta capaz de visualizar a recorrência de estados $x_{i}$ em um espaço de fase. Geralmente, um espaço de fase tem mais de três dimensões, o que não permite que ele seja retratado. Espaços de fase com dimensões mais elevadas (hiperespaço) só podem ser visualizados pela projeção em sub-espaços de duas ou três dimensões. No entanto, a ferramenta de Eckmann et al. (1987) permite investigar a trajetória $m$-dimensional do espaço de fase através de uma representação bidimensional de suas repetições. A recorrência de um estado $i$ em um tempo diferente $j$ é marcada dentro de uma matriz bidimensional quadrada com pontos 1 e 0 (pontos pretos e brancos respectivamente), onde ambos os eixos são eixos de tempo. Esta representação é o próprio Gráfico de Recorrência (GR). Um GR pode ser matematicamente expresso como:

$$
R_{i, j}=\theta\left(\varepsilon-\left\|x_{i}-x_{j}\right\|\right), \quad x_{i} \in \mathbb{R}^{m}, \quad i, j=1 \ldots N,
$$

em que $N$ é o número de estados considerados $x_{i}$, $\varepsilon$ é a distância limite, $\|\cdot\|$ é a norma e $\theta(\cdot)$ é a função de Heaviside. Para estados recor- rentes, isto é, para estados que estejam em uma região de vizinhos em um raio, se introduz a seguinte notação (Marwan et al., 2007): $x_{i} \approx x_{j} \Leftrightarrow R_{i, j} \equiv 1$

O Gráfico de Recorrência é obtido pela plotagem da matriz de recorrência e traçando um ponto para as coordenadas $(i, j)$ se $R_{i, j} \equiv 1$, e um ponto branco se $R_{i, j} \equiv 0$. O GR sempre tem uma linha diagonal principal, a linha de identidade. Além disso, o GR é simétrico no que diz respeito a diagonal principal. A escolha do melhor valor para raio de sondagem tem sido sugerido e defendido na literatura como uma pequena porcentagem do diâmentro máximo do espaço de fase (Mindlin \& Gilmore, 1992).

O propósito inicial de GR é a inspeção visual de trajetórias em espaços de fase de altas dimensões. A visão de um GR dá sugestões sobre a evolução temporal destas trajetórias. A vantagem de GR é que eles também podem ser aplicados a conjuntos de dados curtos e não estacionários.

Os gráficos de recorrência exibem características de grande escala e padrões de pequena escala. Os primeiros padrões de forma denotados por Eckmann et al. (1987) foram a tipologia e a textura. 
A

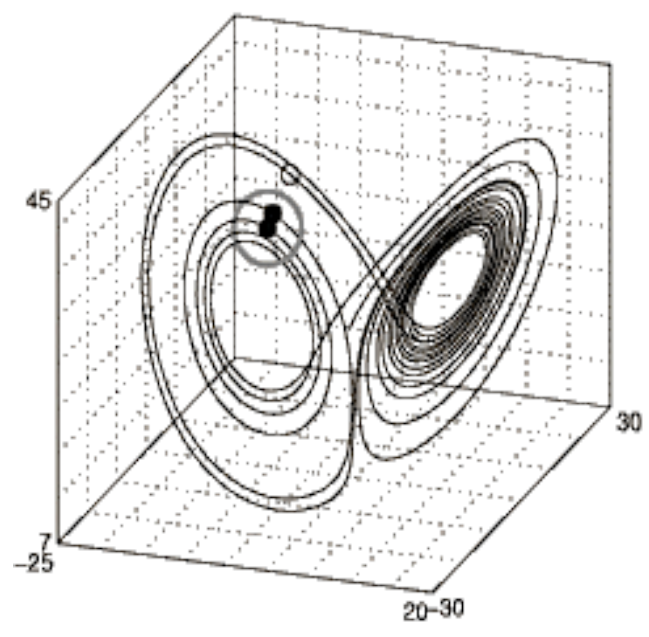

B

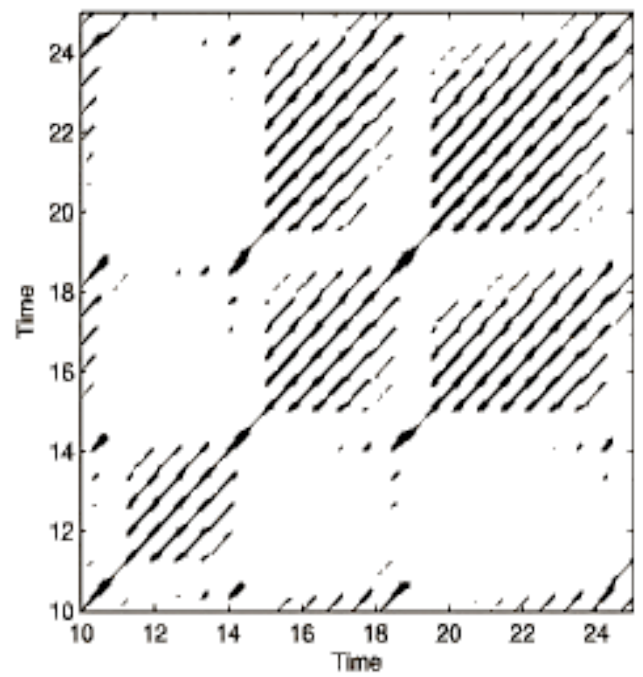

Figura 1 - (A) Segmento da trajetória no espaço de fase do sistema de Lorenz (parâmetros de controle $r=28, \sigma=10, b=03 / 08$; Lorenz, 1963), usando as três componentes x, y e z; e (B) correspondente a sua parcela de recorrência. Um ponto da trajetória de j dentro da vizinhança (círculo cinza em (A)) de um determinado ponto no i é considerado como um ponto de recorrência (ponto preto sobre a trajetória de (A)). Este é marcado com um ponto preto no RP no local (i,j). Um ponto fora da vizinhança (pequeno círculo de (A)) provoca um ponto branco no RP. O raio da vizinhança para o GR é $\varepsilon=5$ (Disponível em $<\underline{\text { http: } / / \text { www.recurrence- }}$ -plot.tk/glance.php> acesso em 12/2011).

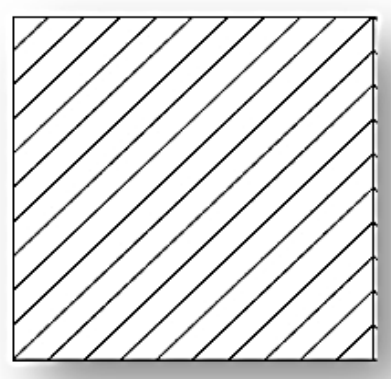

A

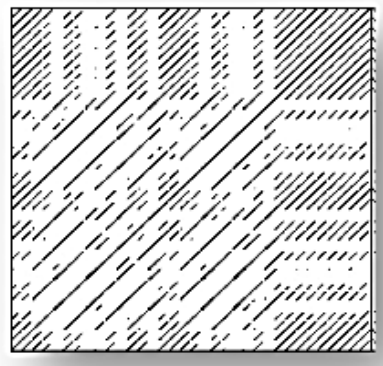

B

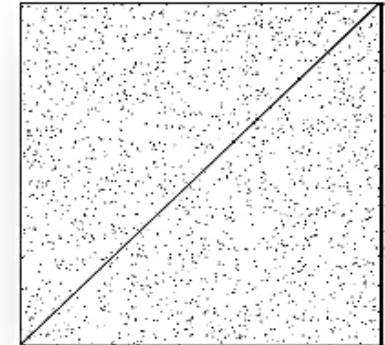

$\mathrm{C}$

Figura 2 - Gráficos de Recorrência de (A) movimento periódico de uma frequência, (B) atrator estranho de Rossler (parâmetros $a=b=0.2$ e c=5.7) e (C) ruído branco (adaptado de Marwan et al., 2007).

A tipologia oferece uma impressão global que pode ser caracterizada como homogênea, flutuante, periódica e desviante. Sistemas homogêneos, típicos de sistemas estacionários e autônomos, possuem tempo de relaxação curto em comparação com o tempo abrangido pelo GR. Um exemplo é o GR de uma série temporal de dados aleatórios. Em sistemas oscilantes o GR contêm diagonais orientadas, estruturas periódicas recorrentes (linhas diagonais, estruturas quadriculadas).

Em sistemas quase periódicos, as distâncias entre as linhas diagonais no GR são diferentes. No entanto, mesmo em sistemas oscilantes cujas oscilações não são facilmente reconhecíveis, os GRs podem ser utilizados a fim de encontrar suas oscilações. Sistemas não estacionários são 
os desvios causados por sistemas com parâmetros de variação lenta. Tal mudança lenta (adiabática) ilumina o canto superior esquerdo e inferior direito do GR. Mudanças abruptas na dinâmica, bem como eventos extremos causam áreas brancas ou bandas no GR.

Uma inspeção mais refinada dos GRs revela estruturas de pequena escala (a textura), que são pontos isolados, linhas diagonais, bem como linhas verticais e horizontais (a combinação de linhas verticais e horizontais, formas retangulares aglomerados de pontos de recorrência). Podem ocorrer pontos de recorrência isolados se os estados são raros, se não persistir por muito tempo ou se eles oscilam muito. No entanto, eles não são um sinal exclusivo do acaso ou de ruído (por exemplo, em mapas de atratores estranhos).

A linha diagonal $R_{i+k, j+k}=1$ (para $k=1 \ldots l$, onde $l$ é o comprimento da linha diagonal) ocorre quando um segmento da trajetória segue paralelo a outro segmento, ou seja, a trajetória visita a mesma região de espaço a fase em momentos diferentes. O comprimento desta linha diagonal principal é determinado pelo tempo de duração semelhante da evolução local dos segmentos de trajetória.

A direção dessas estruturas diagonais pode ser diferente. Linhas diagonais paralelas à linha principal representam trajetórias paralelas seguindo uma mesma evolução temporal. As estruturas diagonais perpendiculares à linha diagonal principal representam o um seguimento de evolução contrária.

A linha vertical (horizontal), $R i \mathrm{j}+\mathrm{k}=1$ (para $\mathrm{k}=1 \ldots \mathrm{v}$, onde $\mathrm{v}$ é o comprimento da linha vertical) marca um período de tempo em que um estado não muda ou muda muito lentamente. Ao que parece, estes estados são presos por algum tempo, comportamento típico de estados laminares (intermitência). Essas estruturas de pequena escala são a base de uma análise quantitativa dos GRs.

\section{Análise da Complexidade}

Embora as estruturas GR possam por si só caracterizar alguns sistemas, dando forma às diferentes tipologias, elas desempenhas um papel fundamental nas quantidades relacionadas ao padrão de pequena escala, a chamada textura. Estas quantidades se baseiam tanto na densidade de recorrência quanto nas quantidades e tamanhos das linhas diagonais e verticais (horizontais).

A análise de complexidade é dada pelas Medidas de Quantificação de Recorrência (MQR), que é um método de análise de dados não lineares que quantifica o número e duração das recorrên- cias de um sistema dinâmico representado por sua trajetória no espaço de fase das variáveis. A análise da MQR foi desenvolvida por Zbilut \& Webber Jr (Zbilut \& Webber Jr., 1992; Webber Jr \& Zbilut, 1994) e estendida com novas medidas de complexidade por Marwan et al. (2002).

\section{MATERIAL E MÉTODOS}

\section{Sítio Experimental e Instrumentação}

A área experimental localiza-se na Reserva Particular do Patrimônio Natural - RPPN SESC Pantanal, município de Barão de Melgaço - MT, distante $160 \mathrm{~km}$ de Cuiabá, Mato Grosso, na Região Centro-Oeste do Brasil onde está instalada uma torre micrometeorológica de $32 \mathrm{~m}$ de altura (16 39'50's; $56^{\circ} 47^{\prime} 50^{\prime \prime} \mathrm{O}$, altitude de $120 \mathrm{~m}$ ).

A torre é rodeada por uma vegetação florestal monodominante de cambará (Vochysia divergens), localmente chamada de cambarazal, em uma faixa contínua de $25 \mathrm{~km}$ de extensão por $4 \mathrm{~km}$ de largura, paralela ao rio Cuiabá (Junk, 2002) e com altura do dossel entre 28 a $30 \mathrm{~m}$. O cambarazal tem apresentado rápido espalhamento nos campos inundados sazonalmente (Arieira \& Nunes da Cunha, 2006).

O solo é GLEISSOLO HÁPLICO Ta Distrófico. O clima é AW segundo Köppen, a temperatura do ar média anual oscila entre $22^{\circ} \mathrm{C}$ e $32^{\circ} \mathrm{C}$ e a precipitação média anual entre 1100 e $1200 \mathrm{~mm}$ com período seco de maio a setembro (Hasenack, 2003).

Tabela 1 - Subdivisão dos dados por mês/ano e características sazonais (período chuvoso e seco) em uma floresta sazonalmente inundada no norte do Pantanal

\begin{tabular}{cc}
\hline Mês / Ano & Sazonalidade \\
\hline Setembro de 2006 & Seco 2006 \\
Fevereiro de 2007 & Chuvoso 2006/07 \\
Agosto de 2007 & Seco 2007 \\
Janeiro de 2008 & Chuvoso 2007/08 \\
Agosto de 2008 & Seco 2008 \\
Janeiro de 2009 & Chuvoso 2008/09 \\
Agosto de 2009 & Seco 2009 \\
Nov/Dez de 2009 & Chuvoso2009/10 \\
\hline
\end{tabular}

Os valores de saldo de radiação, analisados neste trabalho, foram medidos por meio de um saldo radiômetro (Net Radiometer, Kipp \& Zonen Delft, 
Inc., Holland) instalado a $33 \mathrm{~m}$ de altura. Os dados foram coletados a cada 1 segundo e armazenados em médias de 15 minutos em um datalogger (CR 10X, Campbell Scientific, Inc., Ogden, Utah).

Os dados desse trabalho são referentes ao período seco de 2006 até o período chuvoso de 2009/2010, perfazendo um espectro de oito (8) sazonalidades, e aproximadamente quatro (4) anos (Tabela 1).

\section{Métodos}

Neste trabalho, foram utilizadas duas Medidas de Quantificação de Recorrência, a taxa de recorrência (RR) e o determinismo (DET).

A taxa de recorrência (RR) mede a densidade de pontos de recorrência no GR, definido pela equação abaixo.

$$
R R(\varepsilon)=\frac{1}{N^{2}} \sum_{i, j=1}^{N} R_{i, j}(\varepsilon)
$$

A RR não exclui a diagonal principal e, no limite $N \rightarrow \infty$ é a probabilidade de que um estado retorne à sua vizinhança de raio $\varepsilon$ no espaço de fase.

O determinismo $(D E T)$ é a medida de previsibilidade do sistema, definido pela equação abaixo.

$$
D E T=\frac{\sum_{l=l_{\min }}^{N} l P(l)}{\sum_{l=1}^{N} l P(l)}
$$

em que $P(l)$ é o número de diagonais de comprimentol no GR, e $l_{\min }$ é o menor tamanho para uma linha ser considerada uma diagonal.

Os cálculos foram realizados no Matlab ${ }^{\circledR}$, utilizando um pacote definido como TOCSY - Toolbox for Complex Systems pelo Potsdam Institute for Climate Impact Research (PIK) (disponível em <http://tocsy.agnld.uni-potsdam.de $>$ acesso em 27/04/2011).

\section{RESULTADOS E DISCUSSÃO}

A reconstrução do atrator com coordenadas de atraso temporal não é um processo automático, logo é importante a escolha de um $\tau$ apropriado (Baker \& Gollub, 1996). Palú (2008) usou o método da Informação Mútua (Abarbanel et al., 1993; Baker
\& Gollub, 1996) para escolher o melhor tempo de defasagem temporal $(\tau)$ em análise de dados provenientes da torre micrometeorológica na Amazônia Legal em Sinop - MT. Palú (2008) afirma que um tempo de defasagem $\tau=5$ horas pode ser adotado em quaisquer que sejam as análises de séries temporais e para a reconstrução de atratores dos processos envolvidos na interação atmosfera-biosfera daquela floresta, independentemente de qual variável estiver sendo estudada e de que época do ano os dados se referem não existindo diferenciação entre as estações. Assim, definiu-se para a variável uma defasagem temporal $(\tau)$ igual a $5 \mathrm{~h}(\mathrm{~m} \Delta \mathrm{t}=20 \times 15 \mathrm{~min}$, em que $\mathrm{m}$ é um número inteiro e $\Delta \mathrm{t}$ é um intervalo entre cada amostra), visto a baixa correlação entre a série original e as série defasada. Os resultados de Palú (2008) corroboram os de Baker \& Gollub (1996) em que a escolha do parâmetro $\tau$, em situações práticas, pode ser regulado até que os resultados se tornem satisfatórios. Também que uma pequena variação no valor de $\tau$ não é significativa para alterar os resultados obtidos pelo método de análise.

Neste trabalho, também foi calculado o atraso $\tau$ pela função auto-correlação como proposto por Kantz \& Schreiber (1996), encontrando o mesmo valor de $5 \mathrm{~h}$. O melhor espaço de imersão $\mathrm{m}$ para evitar a interferência dos falsos vizinhos foi calculado pelo algoritmo de Grassberger \& Procaccia (1983), assim como o expoente dimensão de correlação (D2), valor aproximadamente igual à dimensão fractal do atrator do sistema.

A estimativa para a dimensão de correlação do balanço de radiação pelo algoritmo de Grassbergue \& Procaccia (1983) foi de $\mathrm{D}_{2 \text { (chuvoso) }}=1,73$ $\pm 0,05$ e $\left.D_{2(\text { seco) }}=1,36 \pm 0,18\right)$ e para a dimensão embutida $m=7$ para as duas sazonalidades (Figura 3 e Figura 4).

Existe um patamar de saturação alcançado em quase todas as análises e observa-se em algumas um pequeno acréscimo na dimensão de correlação, o que pode ser devido a alguma componente não linear no sistema.

Para melhor visualizar e analisar as trajetórias no espaço de fase, verificou-se os seus padrões típicos e as suas ligações com o comportamento específico do sistema, todos os GRs (Figura 5 e Figura 6) foram calculados reconstruindo atratores embutidos em um espaço de fase de 7 dimensões $(\mathrm{m}=7)$, com um atraso $(\tau)$ de 5 horas, usando a norma euclidiana (esfera de sondagem) com $\varepsilon=2$, conforme e Mindlin \& Gilmore (1992).

Houve maior flutuação e maiores médias do balanço de radiação durante o período chuvoso, ocorrendo o oposto durante o período seco. Durante os meses de setembro de 2006, agosto de 

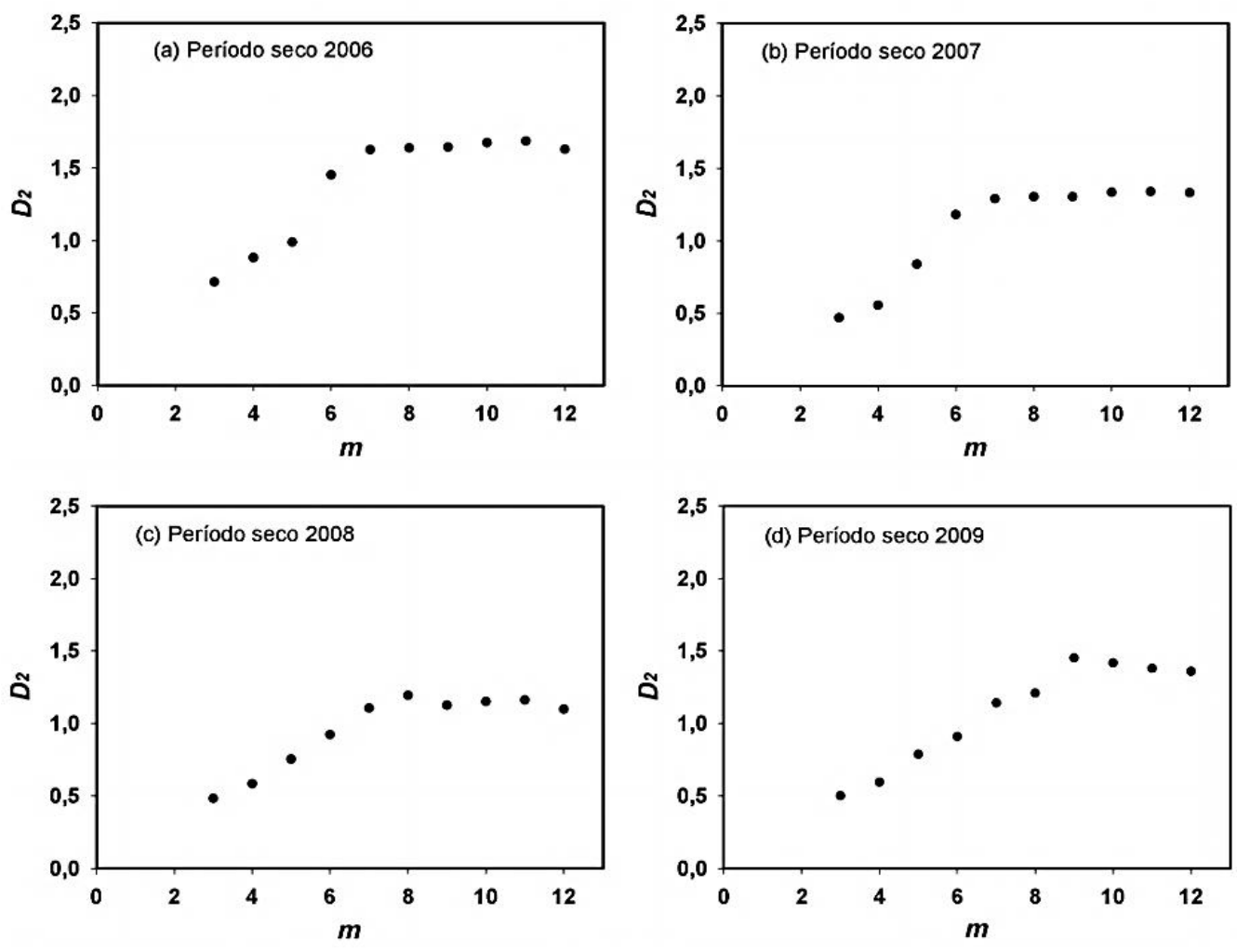

Figura 3 - Estimativa da melhor dimensão de imersão m para o atrator a partir do comportamento da dimensão embutida $m(\mathrm{Rn})$ versus dimensão de correlação $\mathrm{D}_{2}$ referentes aos períodos secos: (a) 2006, (b) 2007, (c) 2008 e (d) 2009, em uma floresta sazonalmente inundada no norte do Pantanal.
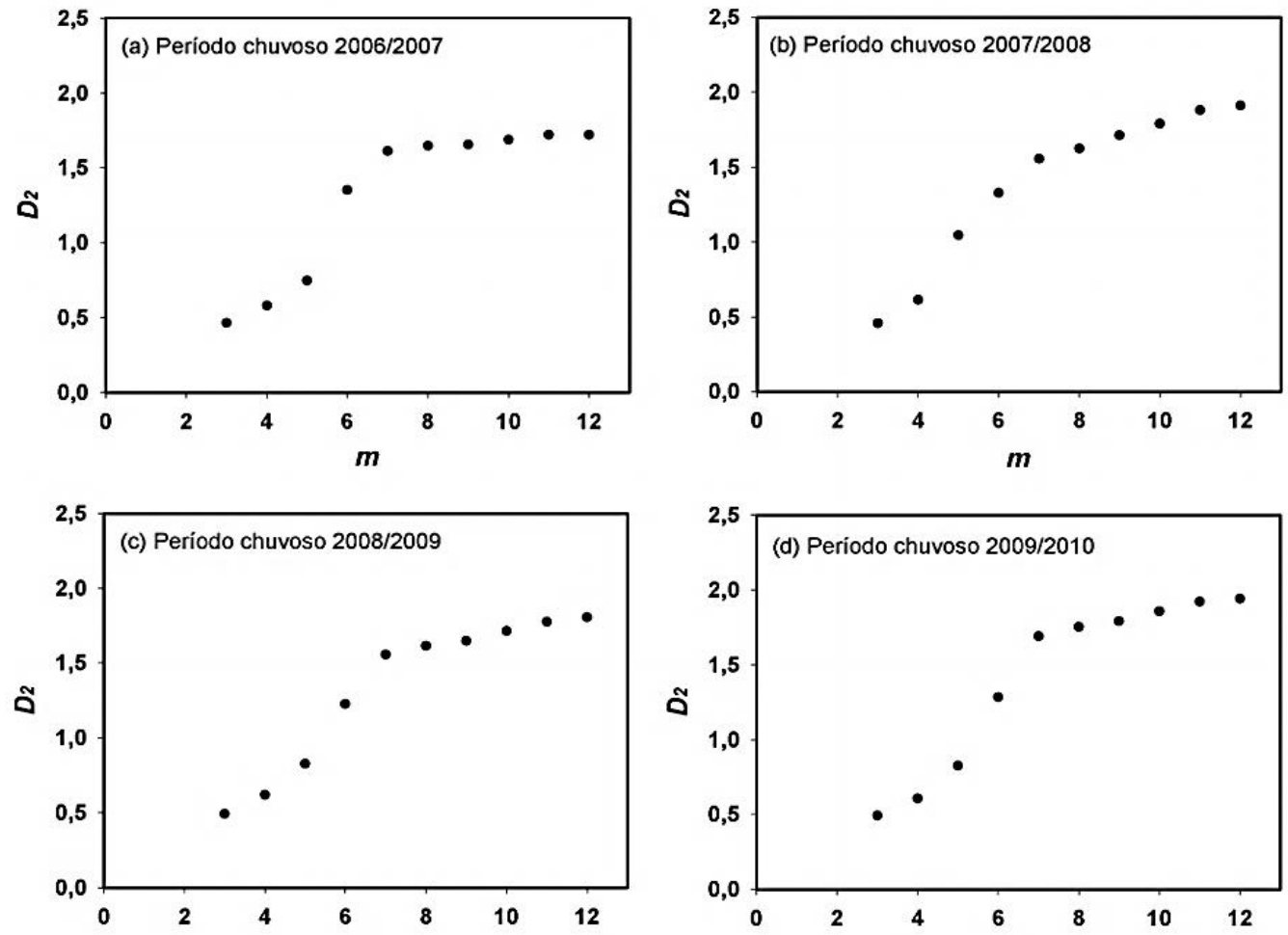

Figura 4 - Estimativa da melhor dimensão de imersão m para o atrator a partir do comportamento da dimensão embutida $m(\mathrm{Rn})$ versus dimensão de correlação $D_{2}$ referentes aos períodos chuvosos: (a) 2006/2007, (b) 2007/2008, (c) 2008/2009 e (d) 2009/2010, em uma floresta sazonalmente inundada no norte do Pantanal. 


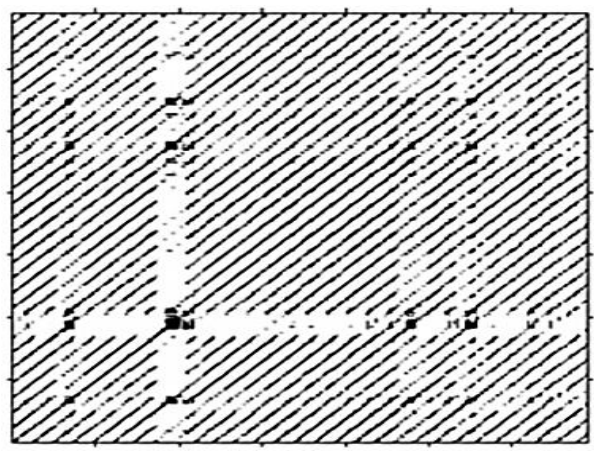

(a)

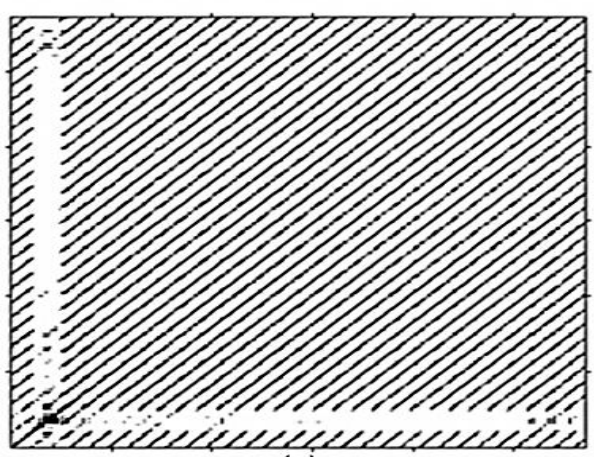

(c)

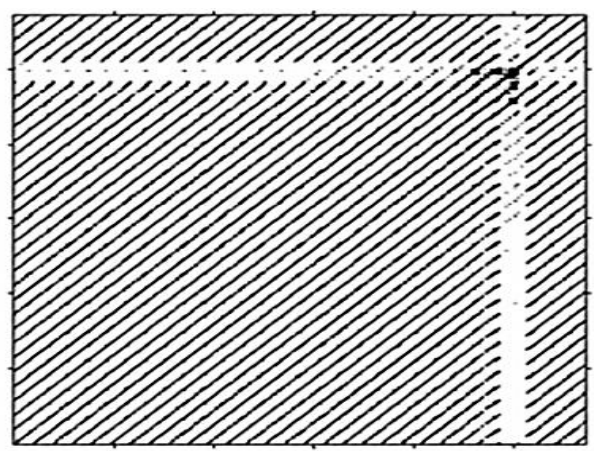

(b)

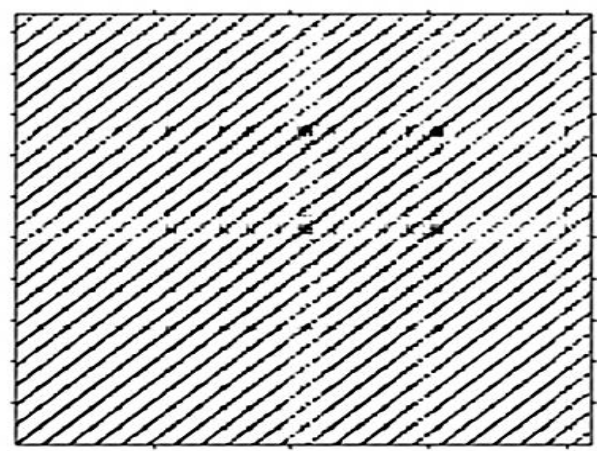

(d)

Figura 5 - Períodos secos. Gráficos de recorrência do balanço de radiação (Rn) referentes à (a) setembro de 2006, (b) agosto de 2007, (c) agosto de 2008 e (d) agosto de 2009, em uma floresta sazonalmente inundada no norte do Pantanal.

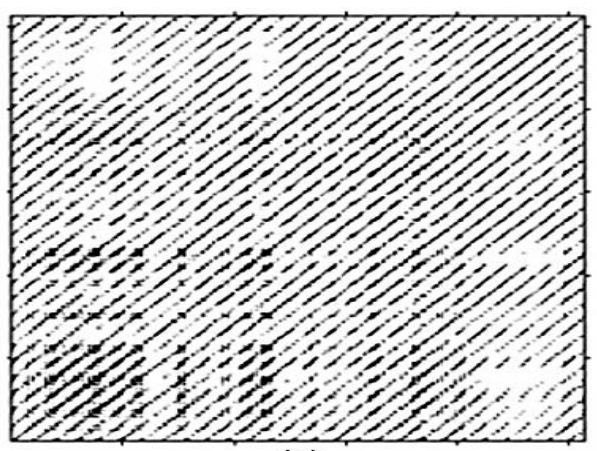

(a)

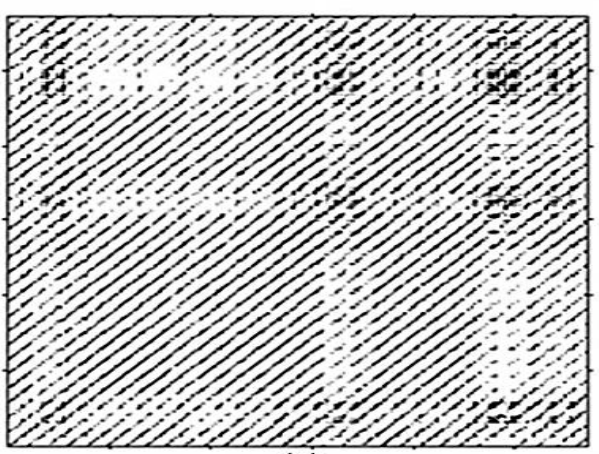

(c)

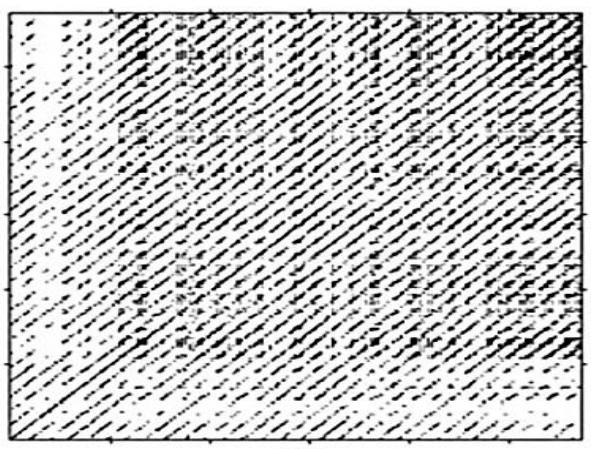

(b)

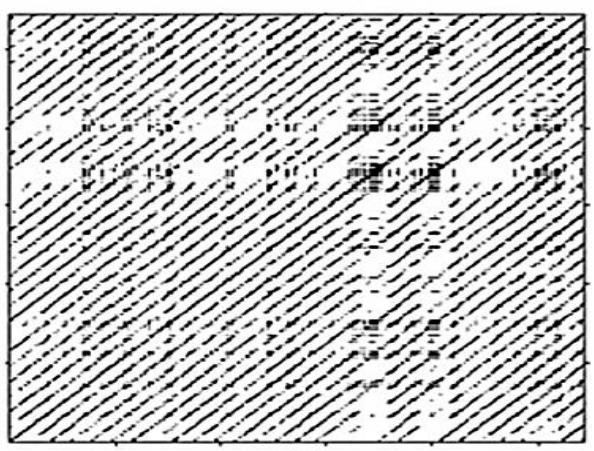

(d)

Figura 6 - Períodos chuvosos. Gráficos de recorrência do balanço de radiação (Rn) referentes à (a) fevereiro de 2007, (b) janeiro de 2008, (c) janeiro de 2009 e (d) novembro e dezembro de 2009, em uma floresta sazonalmente inundada no norte do Pantanal. 
2007, agosto de 2008 e agosto de 2009 (Figura 5) apresentaram minúcias de conjuntos de estados não estacionários (raros ou muito longe do normal), podendo ainda ter ocorrido uma transição de estado (Marwan et al.; 2007). Percebe-se comportamento cíclico pela distância temporal entre os padrões das linhas paralelas que correspondem ao período.

A evolução dos estados é semelhante em diferentes épocas, tendendo a um processo determinista, visto as linhas diagonais paralelas à linha identidade. Breves faixas brancas observadas nas figuras $4 \mathrm{a}, 4 \mathrm{~b}$ e $4 \mathrm{c}$ e levemente em $4 \mathrm{~d}$ também são características de dados não estacionários, ou seja, podem ter ocorridos alguns estados diferenciados, transições de fase no mecanismo de balanço de radiação. Formações de aglomerados (clusters) em todos os gráficos dos períodos secos são indicadores de estados que não mudam, ou mudam muito lentamente no decorrer do tempo, os chamados estados laminares (Zbilut \& Webber Jr., 1992). Esses aglomerados, provavelmente, são relativos à presença marcante de massas de ar frio (frentes frias) que chegam ao Pantanal pelo sul do Brasil. Essas frentes frias, conhecidas como friagens, são enormes massas de ar frio que provocam nevoeiro e costumam ter a duração de 3 a 7 dias, passando a influenciar significativamente a dinâmica atmosférica da região (Webber Jr \& Zbilut, 1994). Mesmo com esses destaques, o padrão dominante dos gráficos de recorrência para os períodos secos é semelhante ao padrão teórico da figura $2 \mathrm{a}$, movimento periódico de uma frequência, mas permeado de intermitências. Houve uma queimada de elevada extensão em 2006 e a fumaça soma-se as causas do padrão do gráfico de recorrência.

Ressalta-se que setembro de 2006 apresenta menor média do Rn, o que pode estar relacionado com o início das queimadas na região, aumentando a quantidade de partículas na atmosfera (Biudes et al., 2009).

Além do processo de absorção, ocorre na atmosfera o processo de difusão da radiação solar, determinada pelos seus diferentes constituintes. Esse processo é responsável por perdas apreciáveis da radiação que retorna para o espaço em virtude das dimensões das partículas determinantes da difusão. Essa difusão específica, determinada por partículas de maiores dimensões, não é dependente do comprimento de onda da radiação, havendo difusão para todos os comprimentos de onda e resultando a cor branca da luz difusa (Reichardt \& Timm, 2008). Esse fenômeno de nevoeiro, que é branco acinzentado, é muito comum no período seco.

Os meses de fevereiro de 2007, janeiro de
2008, janeiro de 2009 e novembro de 2009 apresentam linhas diagonais e estruturas de malha, características de sistemas com mais de duas frequências harmônicas, com períodos dentro de outros períodos. Nessas estruturas de malha (janelas de ciclos dentro de ciclos), em uma inspeção mais próxima, é possível observar estruturas de pequena escala, a sua textura, classificada em pontos isolados, linhas diagonais bem como linhas verticais e horizontais; ainda a combinação de linhas verticais e horizontais, obviamente formas retangulares de grupos de pontos de recorrência. Os descontínuos de pontos seguidos de retas paralelas à linha identidade explicitam o caráter oscilante entre aleatório e periódico dos estados do sistema (Marwan et al.; 2007). É possível perceber faixas brancas características de dados não estacionários provenientes de estados menos frequentes, transições de fase no mecanismo do balanço de radiação. Observa-se ainda que o padrão que mais se destaca são as estruturas paralelas à linha identidade, o que se deve a forçante diária da energia solar.

As Medidas de Quantificação de Recorrência, que quantificam o número e duração das recorrências do sistema dinâmico representado pela sua trajetória no espaço de fase, oscilaram em estados transitórios sazonalmente (Tabela 2).

Tabela 2 - Análise sazonal da taxa de recorrência (RR) e do determinismo (DET) em uma floresta sazonalmente inundada no norte do Pantanal.

\begin{tabular}{ccc}
\hline & RR & DET \\
\hline Seco 2006 & 0,1476 & 0,9521 \\
Chuvoso & 0,1412 & 0,8985 \\
2006/07 & 0,1713 & 0,9916 \\
Seco 2007 & 0,1463 & 0,8824 \\
Chuvoso & 0,1699 & 0,9807 \\
$2007 / 08$ & & \\
Seco 2008 & 0,1486 & 0,9163 \\
Chuvoso & & \\
2008/09 & 0,166 & 0,9688 \\
Seco 2009 & & 0,8857 \\
\hline $\begin{array}{l}\text { Chuvoso } \\
2009 / 10\end{array}$ & 0,1394 & \\
\hline
\end{tabular}

A taxa de recorrência $(R R)$ varia de 0 a 1 , indicando a probabilidade de que um estado retorne à vizinhança de raio $\varepsilon$ no espaço de fase (Webber Jr \& Zbilut, 1994). Os resultados encontrados para 


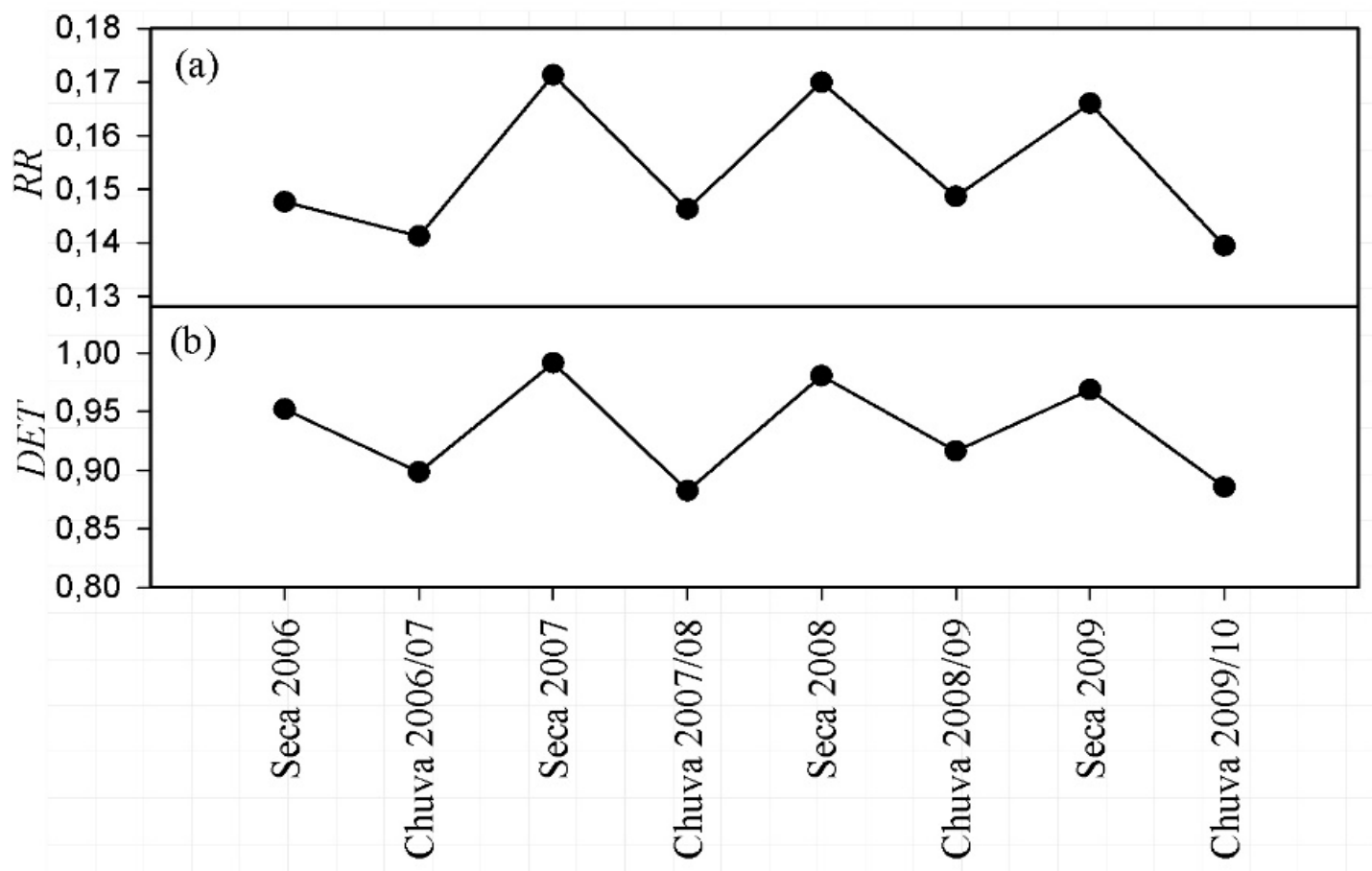

SAZONALIDADE

Figura 7 - Resultados sazonais da (a) taxa de recorrência e do (b) determinismo do saldo de radiação do Pantanal.

RR $(0,1439 \pm 0,0037$ para os períodos chuvosos e $0,1691 \pm 0,0022$ para os períodos secos) indicam que, embora o sistema apresente uma característica senoidal ligado à forçante da radiação solar, as trajetórias no espaço de fase não retornam exatamente à mesma vizinhança em seus ciclos diários (Figura 7).

O determinismo DET é a razão entre o número de pontos pertencentes às diagonais e o número de pontos recorrentes. Logo, quanto mais e maiores forem as diagonais de um GR, mais pares de trechos do atrator se comportam de maneira semelhante, mostrando que uma previsibilidade maior é possível. Esses valores variam de 0 a 1; quando o valor tende a 0 , o sistema é estocástico e quando tende a 1 o sistema é determinístico (previsível) (Marwan et al., 2007). Os resultados encontrados para DET possibilitam afirmar que com um modelo apropriado é possível determinar (ou prever) o saldo de radiação no Pantanal com mais de $89 \%$ de certeza no período chuvoso $(0,8957 \pm 0,0133)$ e com mais de $97 \%$ de certeza no período seco $(0,9733 \pm 0,0147)$. Os valores de DET para o período chuvoso são limitados pela participação da nebulosidade na dinâmica do balanço de radiação; enquanto que muito possivelmente para o período seco sofrem a influência dos aerossóis e emissões por queimadas (Artaxo et al.; 2005, Biudes et al., 2009).

É possível que os resultados obtidos tenham relação com o desmatamento no saldo de radiação das florestas sazonalmente alagadas do Pantanal. Contudo um número maior de observações e de séries temporais mais longas se fazem necessárias para se afirmar com segurança sobre esses possíveis efeitos.

\section{CONCLUSÃO}

As análises dos dados de saldo de radiação quanto às estimativas da dimensão de correlação e da dimensão embutida sugerem a existência de atratores estranhos de baixa dimensão fractal com sensível variação sazonal.

O saldo de radiação no Pantanal segue uma dinâmica periódica acoplada a uma componente não linear complexa. Houve variabilidade sazonal do comportamento dinâmico entre os períodos secos e chuvosos das medidas complexas de taxa de recorrência (RR) e de determinismo (DET). Observou-se que o ecossistema é bastante sensível às mudanças temporais pela presença de intermitências.

Os GRs contribuíram para melhor caracte- 
rizar a complexidade do sistema, exibindo informações importantes sobre sua dinâmica e facilitando a interpretação desses estados em séries temporais.

\section{AGRADECIMENTOS}

Os autores agradecem à Coordenação de Aperfeiçoamento de Pessoal de Nível Superior (CAPES) pelo apoio financeiro; e ao Dr. Norbert Marwan pela caixa de ferramentas CRP para Matlab ${ }^{\circledR}$.

\section{REFERÊNCIAS BIBLIOGRÁFICAS}

ABARBANEL, H. D. I. et al. The analysis of observed chaotic data in physical systems. Reviews of Modern Physics, v.65, n.4, p.1331-1392, 1993.

ARIEIRA, J.; NUNES DA CUNHA, C. Fitossociologia de uma floresta inundável monodominante de Vochysia divergens Pohl (Vochysiaceae), no Pantanal Norte, MT, Brasil. Acta Botanica Brasilica, v.20, p.569-580, 2006.

ARTAXO, P. et al. Química atmosférica na Amazônia: a floresta e as emissões de queimadas controlando a composição da atmosfera amazônica. Acta Amazônica, v.35, n.2, p.185-196, 2005.

BAKER, G. L.; GOLLUB, J. P. Chaotic dynamics: an introduction. New York, USA: Cambridge University Press; 1996. 256p.

BIUDES, M. S. et al. Estimativa do balanço de energia em cambarazal e pastagem no norte do pantanal pelo método da razão de Bowen. Revista Brasileira de Meteorologia, v.24, n.2, p.135-143, 2009.

CAMPANHARO, A.S.L.O. et al. Searching chaos and coherent structures in the atmospheric turbulence above the Amazon forest. Philosophical Transactions of the Royal Society A, v.366, p.579-589, 2008.

COSTA, M. H.; PIRES, G. F. Effects of Amazon and Central Brazil deforestation scenarios on the duration of the dry season in the arc of deforestation. International Journal of Climatology, v.30, p.1970-1979, 2010.

DINIZ, G.; FONSECA, M.; JR CAMPELO, J. H. Análise harmônica do regime de precipitação em duas localidades da baixada cuiabana. Biomatemática, v.18, p.37-48, 2008.

ECKMANN, J. P.; KAMPHORST, S. O.; RUELLE, D. Recurrence plots of dynamical systems. Europhysics
Letter, v.4, n.9, p.973-977, 1987.

FRAEDRICH, K. Estimating the dimensions of weather and climatic attractors. Journal of the Atmospheric Sciences, v.43, p.419-432, 1986.

Gallego, M. C.; Garcia, J.A.; Cancillo, M. L. Characterization of atmospheric turbulence by dynamical systems techniques. Boundary-Layer Meteorology, v.100, p.375-392, 2001.

GOMES, A. G.; VARRIALE, M. C. Modelagem de Ecossistemas: Uma Introdução. Editora da Universidade Federal de Santa Maria: Santa Maria, 2004. 503p.

GRASSBERGER, P.; PROCACCIA, I. Characterization of strange attractors. Physical Review Letters, v.50, n.5, p.346-349, 1983.

HASENACK, H.; CORDEIRO, J. L. P.; HOFMANN, G. S. O Clima da RPPN SESC-Pantanal. Porto Alegre, UFRGS, 27 p., 2003.

INTERGOVERNMENTAL PANEL ON CLIMATE CHANGE - IPCC. 2007. Synthesis Report. Contribution of Working Groups I, II and III to the Fourth Assessment Report of the Intergovernmental Panel on Climate Change. Geneva, Switzerland, 2007. 104p.

JUNK, W. J. Long-term environmental trends and the future of tropical wetlands. Environmental Conservation, v.29, n.4, p.414-435, 2002.

JUNK, W. J.; NUNES DA CUNHA, C. Pantanal: a large South American wetland at a crossroads. Ecological Engineering, v.24, p.391-401, 2005.

KANTS, H.; SCHREIBER, T. Nonlinear time series analysis. Cambridge University Press: Cambridge, UK, 2004. 369p.

LAPOLA, D. M.; SCHALDACH, R.; ALCAMO, J. Impacts of climate change and the end of deforestation on land use in the Brazilian Legal Amazon. Earth Interactions, v.15, n.16, p.1-19, 2011.

LEWIS, S. L. et al. The 2010 Amazon Drought. Science, v.331, p.554, 2011

LORENZ, E. N. Deterministic nonperiodic flow. Journal of Atmospheric Sciences, v.20, p.130-141, 1963.

LORENZ, E. N. Dimension of weather and climate attractors. Letters to Nature, v.353, p.241-244, 1991. 
MARWAN, N. et al. Recurrence plot based measures of complexity and its application to heart rate variability data, Physical Review E, v.66, p.026702(1-8), 2002.

MARWAN, N. et al. Recurrence plots for the analysis of complex systems. Physics Reports, v.438, p.237-329, 2007.

McCANN, K. V. The diversity-stability debate. Nature, v.405: p.228-233, 2000.

McClain, M. E. The Ecohydrology of South American Rivers and Wetlands. IAHS Press: Wallingford, UK. 2008. 209p.

\section{MILLENNIUM ECOSYSTEM ASSESSMENT.}

Ecosystems and Human Well-Being: Wetlands and Water Synthesis. World Resources Institute, Washington, DC, 2005. 80p.

MINDLIN, G. M.; GILMORE, R. Topological analysis and synthesis of chaotic time series, Physica D Nonlinear Phenomena, v.58 n.1-4, p.229-242, 1992.

MOTTA, R. S.; HARGRAVE, J.; LUEDEMANN, GUTIERREZ, M. B. S. Mudança do Clima no Brasil: aspectos econômicos, sociais e regulatórios. Secretaria de Assuntos Estratégicos - SAE. Instituto de Pesquisa Econômica Aplicada - IPEA: Brasília, DF, 2011. 438p.

NICOLIS, C.; NICOLIS, G. Is there a climatic attractor? Nature, v.311, p.529-532, 1984.

NICOLIS, G., PRIGOGINE, I. Exploring complexity: an introduction. W. H. Freeman and Company: Nova York, USA, 1998. 312p.

NOBRE, C. A. et al. Observations of the atmospheric boundary layer in Rondônia. In: GASH, J. H. C. et al. (Eds.) Amazonian Deforestation and Climate. J. M. Wiley and Sons, 1996. p. 413-424.

OMETTO, J.C. Bioclimatologia vegetal. São Paulo: Ed. Agronômica Ceres, 1981. 440p.

PALÚ, A. E. R. Determinação do tempo de defasagem mais adequado para análise de séries temporais de variáveis microclimatológicas medidas numa floresta de transição no norte de Mato Grosso. 2008. 38p. Dissertação (Mestrado em Física Ambiental) - Instituto de Física, Universidade Federal de Mato Grosso, Cuiabá.

PEITGEN, H.; JORGENS, H.; SAUPE, D. Chaos and fractals: new frontiers of science. New York: SpringerVerlag, 2004. 864p.
PEREIRA, R. A.; ANGELOCCI, L. R.; SENTELHAS, P. C. Agrometeorologia: fundamentos e aplicações práticas. Guaíba, Brasil: Agropecuária; 2002. 478p.

POVEDO-JARAMILLO, G.; PUENTE, C. E. Strange attractors in atmospheric boundary-layer turbulence. Boundary-Layer Meteorology, v.64, p.175-197, 1993.

REICHARDT, K.; TIMM, L. C. Solo, planta e atmosfera: conceitos, processos e aplicações. Manole Ltda: Barueri, Brasil, 2004. 478p.

RUELLE, D., TAKENS, F. On the nature of turbulence. Communications in Mathematical Physics, v.20, p.167192, 1971.

SILVA, J. S. V.; ABDON, M. M. Delimitação do Pantanal Brasileiro e suas sub-regiões. Pesquisa Agropecuária Brasileira, v.33, p.1703-1711, 1998.

SOUZA, G.; BUCKERIDGE, M. Sistemas complexos: novas formas de ver a botânica. Revista Brasileira de Botânica, v.27, n.3, p.407-419, 2004.

TAKENS, F. Detecting strange attractors in turbulence. Lecture Notes in Mathematics, v.898, p.366-381, 1981.

WALDROP, M. M. Complexity, the emerging science at the edge of order and chaos. Touchstone, New York, 1992. 380p.

WEBBER JR., C. L.; ZBILUT, J. P. Dynamical assessment of physiological systems and states using recurrence plot strategies. Journal of Applied Physiology, v.76, n.2, p.965-973, 1994.

XIN, L.; FEI, H.; GANG, L. Characteristics of chaotic attractors in atmospheric boundary layer turbulence. Boundary-Layer Meteorology, v.99, p.335-345, 2001.

ZBILUT, J. P.; WEBBER JR., C. L. Embeddings and delays as derived from quantification of recurrence plots. Physics Letters A, v.171, n.3-4, p.199-203, 1992. 